Research Article

\title{
Passive Radar Source Localization Based on Cuckoo Search NVTDOA
}

\author{
Gang Niu $\mathbb{D}^{1,},{ }^{1,2}$ Jie Gao, ${ }^{1}$ and Tai-hang Du $\mathbb{D}^{1}$ \\ ${ }^{1}$ School of Artificial Intelligence, Hebei University of Technology, Tianjin 300401, China \\ ${ }^{2}$ Institute of Mechanical Technology, Shijiazhuang 050000, China \\ Correspondence should be addressed to Gang Niu; 201412501001@stu.hebut.edu.cn
}

Received 11 November 2019; Revised 2 February 2020; Accepted 10 February 2020; Published 14 March 2020

Academic Editor: Giorgio Montisci

Copyright (c) 2020 Gang Niu et al. This is an open access article distributed under the Creative Commons Attribution License, which permits unrestricted use, distribution, and reproduction in any medium, provided the original work is properly cited.

In order to meet the requirement of passive radar source localization in electronic warfare, the concept of the virtual time differences of arrival (VTDOA) is proposed by taking advantage of the characteristics of the same UAV in different positions at different times and the periodic rotation of radar pulse signal. The VTDOAs are the passive localization information defined as the time differences of the radar pulse transmission from the radar position to different virtual receivers. Firstly, a nonlinear VTDOA (NVTDOA) localization model is constructed. Moreover, sufficient conditions for accurately calculating the periodic integers in the model are analyzed, and the observability conditions of the localization model determined are deduced. Secondly, the convergence solution of the NVTDOA localization equation is obtained by Cuckoo search algorithm; thus, passive radar source localization is realized. Finally, the performance of the proposed method is verified by comparing with the existing methods.

\section{Introduction}

Passive localization is a fundamental problem that usually accomplishes localization task using the radiation signals of the target without radiating electromagnetic wave signals outwards, which has been widely used in many fields such as radar [1], sonar [2], sensor network [3], and wireless communication [4]. The passive localization method belongs to a two-step localization system. In the first step, some signal parameters in the target source transmission are extracted, such as received signal strength [5], time difference of arrival (TDOA) [6, 7], and angle of arrival (AOA) $[8,9]$. In the second step, the target position is estimated by solving a set of nonlinear localization equations.

For a long time, the passive localization method based on TDOA has been an important research topic [10]. For noncooperative radiation sources, it is particularly important to study TDOA localization technology for the single station because the time of signal emission is unknown. There are three main calculation methods of TDOA for noncooperative radiation sources. The first is to take the rising edge of the first pulse as the arrival time [11]. The pulse amplitude jump points from scratch are chosen as the reference point of the method, so it is easier and more accurate to determine the reference point.
However, the signal amplitude of the first pulse is small, and the estimation error becomes larger when the pulse loss is more serious or the amplitude is smaller. The second is to take the average arrival time of all received pulses as the arrival time of radar signals [12]. The method has high stability but high computational complexity. If the received pulses overlap, the estimated performance will deteriorate. The third is to select the arrival time of the pulse with the highest amplitude in the main lobe pulse as the receiving time of radar signal [13]. When the main lobe beam is narrow, the arrival time will have higher stability. But if the main lobe beam is wider, there will be more maximum amplitude values in the pulse, which will degrade the estimation performance. In order to overcome the shortcomings of the above TDOA estimation, a norm approximation method was proposed by Wan [14]. The approximate mathematical expression of the main lobe beam pattern of the radar antenna is obtained at the receiving end, and the time point corresponding to the maximum value is used as the arrival time of radar signal to complete the estimation of VTDOA for radar signal.

With the more and more extensive application of the unmanned aerial vehicle (UAV) in battlefield, there are some related research studies on the algorithm of using a UAV receiver as moving single station to locate a radar emitter. A 
passive location method of pulse information sources in the battlefield by using a single UAV was proposed by Nickens and Darko [15]. The method takes the rising time of pulse signals received by the UAV as the signal arrival time and builds localization equations based on UAV's movement trajectory. However, when the pulse amplitude of the source is small or far away, the judgment error of the receiver on the rising moment of the pulse will be larger, and the localization accuracy will be worse. Kim et al. [16] proposed a way for a single drone to use a known database to provide rough location services for targets in the battlefield when the satellite positioning system is not working properly. To improve the performance of the location system in scenarios with a small number of receivers or when there are technical restrictions to deploy them in LoS condition, in reference [17-20], a localization algorithm based on time difference of arrival (TDOA) virtual sensors is proposed, where the reflection points are regarded as TDOA virtual sensors. And the algorithm is applied to localization of UAV in urban scenarios, ground vehicles, emitters, and RF emitters. Based on this, Hao et al. [21], Wan [14], and Zhu et al. [22] put forward a radar radiation source localization method based on passive synthetic aperture antenna array. The method utilizes the characteristics of a single-station small-load UAV in different positions at different times and the periodic rotation characteristics of radar pulse signals and uses VTDOA to complete the localization task. However, the observability of the localization model is not discussed, and the applicable conditions of the model are not analyzed in [14, 21, 22]. Moreover, it is assumed that the two radar signal waves received by the UAV are the same plane waves to obtain the closed solution of the positioning equation, and the radar rotation speed at the first received signal is used to calculate the radar antenna rotation time difference. Therefore, if the UAV runs at a high speed or the localization time difference is long, the localization accuracy of the target radar will become very poor.

For the deficiency of the above algorithm, based on the motion characteristics of UAV and the periodic rotation characteristics of radar pulse signal, a new method of passive localization for the radar emitter based on Cuckoo search NVTDOA is proposed. In conclusion, the Cuckoo search NVTDOA method has made contributions in two aspects compared with the literature. First, the NVTDOA model is not sensitive to the speed of UAV or the localization time difference, so that it has more applicability. However, the approximate calculation of the rotation time difference in the VTDOA model makes its accuracy more sensitive to these, so VTDOA is only applicable to the positioning of UAV at low speed and short stroke. Second, the Cuckoo search algorithm is used instead of the closed-form solution algorithm to solve the localization equation, which finally improves the localization accuracy of the algorithm. In Section 2, the traditional VTDOA localization model is introduced. In Section 3, first, the shortcomings of the VTDOA localization model are analyzed in the first paragraph, so an improved NVTDOA localization model is proposed considering the integral characteristics of radar antenna rotation time difference. Second, the NVTDOA localization model is described according to the traditional two-step localization method. The first step, the derivation of the NVTDOA localization model, is given in Section 3.1, and the second step, the estimation method of virtual time difference in the NVTDOA localization model, is introduced in Section 3.2. Therefore, it provides a localization model for the construction of the following localization equation. In Section 4, the observability of the NVTDOA model is introduced to verify the rationality of the model, and the theoretical basis is provided for the approximate calculation of the localization equationin the followi. Based on this, the construction and solution of the location equation are presented in Section 5. To realize the location of a radar emitter, the location models of two flight segments are established in Section 5.1, and the two models are combined to generate the location equations. According to the nonlinear characteristics of the equations, in Section 5.2, the Cuckoo search algorithm is proposed to solve the equations and finally realize the location of the radar emitter. In Section 6, VTDOA and NVTDOA are used to analyze the location of three different passive radars to verify the high accuracy and antinoise of the NVTDOA algorithm. In Section 7, the theoretical derivation and simulation are summarized and analyzed.

\section{VTDOA Localization Model}

The angular velocity of the target radar antenna rotating uniformly in counterclockwise direction is $\Omega$, and the periodic rotation angle is $\Pi$. Taking the $t_{i}$ moment of the UAV as the origin, a simple motion model of a UAV and the target radar is shown in Figure 1. The angle between the main lobe pulse direction of the far-field radiator and the UAV is $\varphi_{i}$. If the UAV moves in a straight line, it can be regarded as a constant.

Assuming that the radar rotation period $T=\Pi / \Omega$, which can be measured, the UAV receives the radar main lobe pulse signal at position $S_{i}$ at time $t_{i}$, and after a period of time, the UAV moves to position $S_{i+1}$ at time $t_{i+1}$ and receives the radar main lobe pulse signal. Let $P$ be the shortcut point of radiation source $S_{0}$ from the UAV track. The length from $S_{0}$ to $P$ is $D$, and the length from $S_{i}$ to $P$ is $L$. The distance from the initial point $S_{i}$ of the UAV to $S_{0}$ is $d_{i}$ and that from $S_{i+1}$ to $S_{0}$ is $d_{i+1}$. Similarly, the distance from $S_{i+2}$ to $S_{0}$ is $d_{i+2}$. The distance from location $S_{i+1}$ to $S_{i}$ is $l_{i}$, that from $S_{i+2}$ to $S_{i+1}$ is $l_{i+1}$, and so on. Of these quantities, $l_{i}$ and $l_{i+1}$ are known quantities. It can be seen from Figure 1 that, for the fixed radiation source $S_{0}$, it can be characterized by $X=\{D, L\}^{T}$. Then, the measurement equation of the variables to be solved can be written as follows:

$$
\Psi_{i+1, i}=t_{i+1}-t_{i}=\left(\frac{\left(d_{i+1}-d_{i}\right)}{c}\right)+\Delta \psi+K_{i+1, i} T+\Delta t_{i+1, i}
$$

where $c=3.0 \times 10^{8} \mathrm{~m} / \mathrm{s}$, which is the propagation speed of radar radio waves. $\Delta t_{i+1, i}$ is the difference of TOA measurement noise between two locations of UAV, which is modeled as Gaussian white noise with zero mean value and standard deviation of $\sigma_{t}$. $K_{i+1, i}$ represents the integer of radar rotation period, and it means that the time interval between two UAV locations is $K_{i+1, i}$ radar period. $\Delta \psi$ is the rotation time difference of the radar antenna from $S_{i}$ to $S_{i+1}$. 


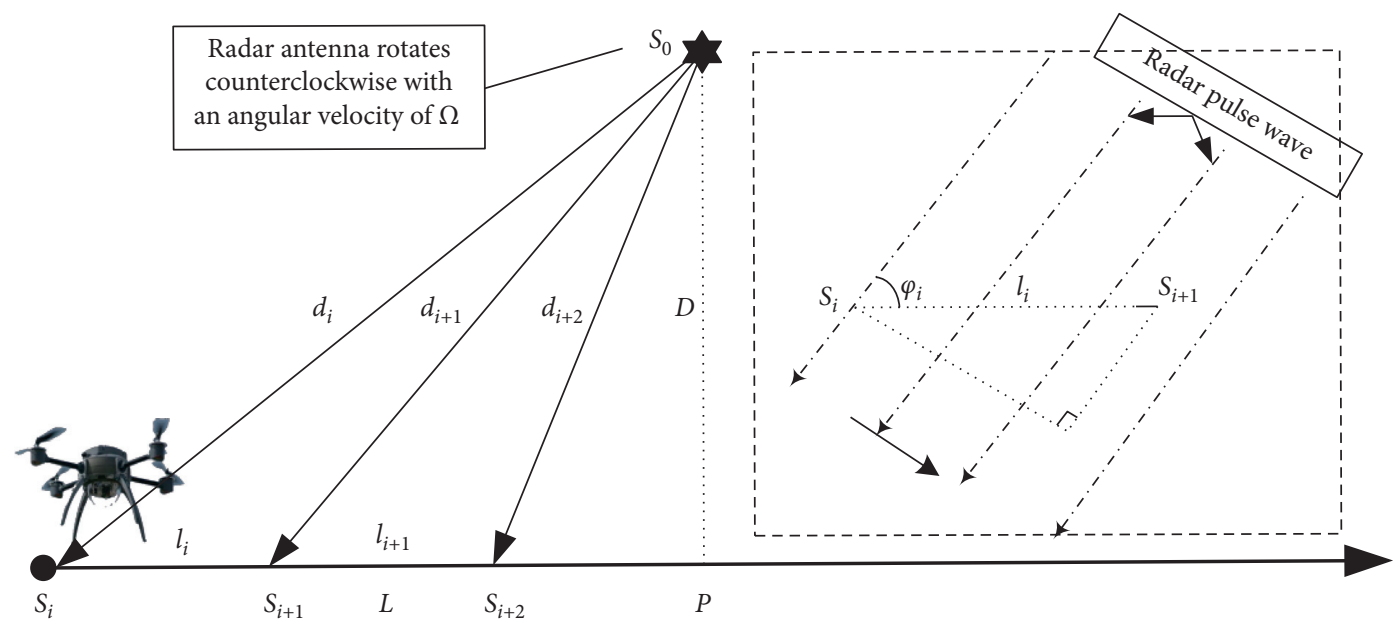

Figure 1: Simple motion model of a UAV and the target radar.

In the VTDOA localization model, it is assumed that the linear velocity of the radar antenna during the rotation from $S_{i}$ to $S_{i+1}$ is the same as

$$
v_{r i}=\Omega \cdot d_{i}
$$

So

$$
\Delta \psi=\frac{l_{i} \sin \varphi_{i}}{v_{r i}}=\frac{l_{i} \sin \varphi_{i}}{\Omega d_{i}} .
$$

Then, the VTDOA localization model is that

$$
\operatorname{VTDOA}_{i+1, i}=\frac{d_{i+1}-d_{i}}{c}+\frac{l_{i} \sin \varphi_{i}}{\Omega d_{i}}+K_{i+1, i} T+\Delta t_{i+1, i} \text {. }
$$

\section{NVTDOA Localization Model}

3.1. NVTDOA Localization Model Based on Rotation Time Difference of Radar Antenna. In [14, 17, 18], in order to find the closed solution of the localization equation, it is assumed that the two radar signal waves received by UAV are the same plane waves. The rotation time difference of the radar antenna is calculated by using the radar rotation speed at the first received signal. Therefore, if the UAV runs at a high speed or the localization time difference is long, the localization accuracy of the target radar will become very poor. So, in this paper, an algorithm is presented to obtain the time difference of the radar antenna rotation based on definite integration of the rotation angle.

As shown in Figure 2, $\Delta \psi$ can be obtained by integrating the rotation angle $\theta$ of the radar antenna. The rotation time difference of the radar from $S_{\theta}$ to any small angle $\Delta \theta$ is $\Delta \psi_{\theta}$; then

$$
\Delta \psi_{\theta}=\left(\frac{\Delta x}{\left(\Omega d_{\theta}\right)}\right)
$$

So $\Delta \psi$ can be expressed as

$$
\Delta \psi=\int_{0}^{\theta_{i+1, i}} \Delta \psi_{\theta}=\int_{0}^{\theta_{i+1, i}} \frac{\Delta x}{\left(\Omega d_{\theta}\right)} .
$$

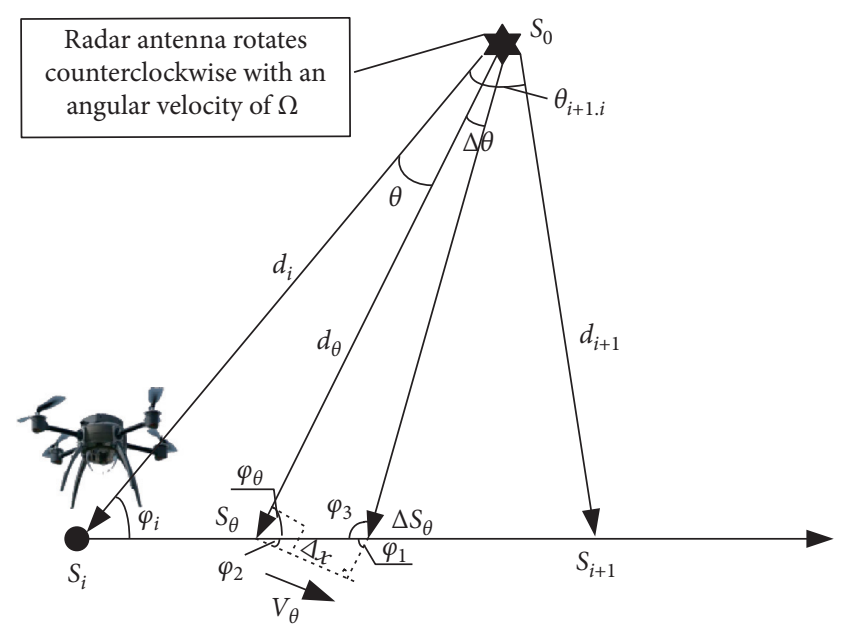

FIGURE 2: The movement process of a UAV from $S_{i}$ to $S_{i+1}$.

According to the sine theorem, $\Delta x$ can be obtained by

$$
\frac{d_{\theta}}{\sin \varphi_{3}}=\frac{\Delta x / \cos \varphi_{2}}{\sin \Delta \theta}
$$

where $\varphi_{2}=\left(\pi /\left(2-\varphi_{\theta}\right)\right), \varphi_{\theta}=\varphi_{i}+\theta, \varphi_{3}=\pi-\Delta \theta-\varphi_{1}$, and $\varphi_{1}=\varphi_{\theta}$; then

$$
\begin{aligned}
\Delta x & =\frac{d_{\theta} \cos \varphi_{2} \sin \Delta \theta}{\sin \varphi_{3}}=\frac{d_{\theta} \cos \left((\pi / 2)-\varphi_{i}-\theta\right) \sin \Delta \theta}{\sin \left(\pi-\Delta \theta-\varphi_{i}-\theta\right)} \\
& =\frac{d_{\theta} \sin \left(\varphi_{i}+\theta\right) \sin \Delta \theta}{\sin \left(\Delta \theta+\varphi_{i}+\theta\right)} .
\end{aligned}
$$

Substituting equation (8) into equation (5) yields

$$
\Delta \psi_{\theta}=\frac{d_{\theta} \sin \left(\varphi_{i}+\theta\right) \sin \Delta \theta}{\sin \left(\Delta \theta+\varphi_{i}+\theta\right) \Omega d_{\theta}}=\frac{\sin \left(\varphi_{i}+\theta\right) \sin \Delta \theta}{\Omega \sin \left(\Delta \theta+\varphi_{i}+\theta\right)},
$$

where $\Delta \theta$ is an infinitesimal amount; then, $\sin \Delta \theta \approx \Delta \theta$, $\sin \left(\Delta \theta+\varphi_{i}+\theta\right) \approx \sin \left(\varphi_{i}+\theta\right)$, and equation (9) can be reduced to 


$$
\Delta \psi_{\theta}=\left(\frac{\Delta \theta}{\Omega}\right)
$$

Substituting equation (10) into equation (6), we have

$$
\Delta \psi=\int_{0}^{\theta_{i+1, i}} \frac{\Delta \theta}{\Omega}=\int_{0}^{\theta_{i+1, i}} \frac{1}{\Omega} \mathrm{d} \theta
$$

where $\theta_{i+1, i}=\cos ^{-1}\left(\left(d_{i}^{2}+d_{i+1}^{2}-l_{i}^{2}\right) /\left(2 d_{i} d_{i+1}\right)\right)$; then

$$
\Delta \psi=\frac{\theta_{i+1, i}}{\Omega}=\frac{\cos ^{-1}\left(\left(d_{i}^{2}+d_{i+1}^{2}-l_{i}^{2}\right) / 2 d_{i} d_{i+1}\right)}{\Omega} .
$$

Substituting equation (12) into equation (1) yields

$$
\Psi_{i+1, i}=t_{i+1}-t_{i}=\left(\frac{\left(d_{i+1}-d_{i}\right)}{c}\right)+\left(\frac{\theta_{i+1, i}}{\Omega}\right)+K_{i+1, i} T+\Delta t_{i+1, i} .
$$

3.2. Estimation Method of NVTDOA. When the radar system conducts periodic scanning, assuming that the UAV starts to collect the data at time $t_{0}$, the UAV will not always be within the range of radar antenna fan beam irradiation. At the ith rotation period of radar, with the antenna rotating, the UAV will enter the range of the antenna main lobe fan beam at time $t_{i}$ and leave the main lobe irradiation area after receiving $N_{P}$ radar main lobe pulses continuously. And the center time of the $k$ th main lobe pulse received in the $i$ th rotation period is $t_{i, k}$. Under the influence of beam gain directionality $G(t), N_{P}$ received pulse signals have different amplitudes. So the baseband signal waveform of the radar radiation source pulse signal received by UAV is shown in Figure 1 , and PRI $=(1 / \mathrm{PRF})$ which is the pulse time interval, where PRF is the radar pulse repetition frequency. So the baseband signal of the linear frequency modulated (LFM) radar pulse received by the UAV can be expressed as follows [22]:

$$
x(t)=P_{T} \cdot G(t) \cdot G_{R} \cdot a(t) \cdot e^{j \varphi_{c}}+\xi(t),
$$

where $\xi(t)$ is additive random noise, $P_{T}$ is the transmitting power of the radar antenna, $G_{R}$ is the transceiver gains, and $\varphi_{c}$ is the initial phase of the signal.

As shown in Figure 3, the aircraft will receive $N_{P}$ main lobe pulses in each rotation cycle of the radar. It is necessary to mine the hidden useful information from the received signal for radar location. The hidden information used in the paper is defined as NVTDOA. NVTDOA is defined as the difference of transmission time required for the radar signal to reach the same receiver at two different positions. In this paper, NVTDOA is represented by $\Psi_{j, i}$, and $t_{i}$ and $t_{j}$ represent the initial time for the same UAV to enter the $i$ th and $j$ th scanning periods of the main lobe of the radar antenna, respectively. So

$$
\Psi_{j, i}=t_{j}-t_{i}
$$

In practical application, it is difficult to accurately estimate $t_{i}$ and $t_{j}$ by $x(t)$ of equation (14). As shown in Figure 3 , let $\Psi_{j, i}=t_{j, \max }-t_{i, \max }$, where $t_{i, \max }$ and $t_{j, \text { max }}$ are the peak times of $G(t)$ of the $i$ th and $j$ th rotation period, respectively. In order to improve the estimation accuracy of NVTDOA, the estimation method of $t_{i, \max }$ and $t_{j \text {, max }}$ in this paper is referred to [21], which will not be described here.

\section{Observability Analysis of Localization Model}

The localization model is obtained in the previous section; the next problem is in which case the equation is solvable, namely, the observability problem of the model $[23,24]$. In this paper, the observability conditions of the localization model are obtained by identifying the parameters of two systems.

\subsection{Calculation Conditions for Integers of Radar Rotation Period}

4.1.1. Calculation Conditions with the Radar Rotation Period Known. When the radar rotation period is known, the period integer can be calculated by the following equation, taking $S_{i}$ and $S_{i+1}$ as examples:

$$
K_{i+1, i}=\operatorname{round}\left(\frac{\left(t_{i+1}-t_{i}\right)}{T}\right)
$$

where round(.) denotes the rounding integer operation. Substituting equation (13) into equation (16) yields

$$
K_{i+1, i}=\operatorname{round}\left(\left(\frac{\left(d_{i+1}-d_{i}\right)}{(c T)}\right)+\left(\frac{\theta_{i+1, i}}{\Pi}\right)+K_{i+1, i}+\left(\frac{\Delta t_{i+1, i}}{T}\right)\right) \text {. }
$$

Sufficient conditions for accurate estimation which can be obtained by equation (17) are as follows:

$$
\begin{aligned}
& \text { Condition (1a): }\left(\left(d_{i+1}-d_{i}\right) /(c T)\right)+\left(\left(\Delta t_{i+1, i}\right) / T\right) \approx 0 \\
& \text { Condition (2a): } \theta_{i+1, i}<0.5 \Pi
\end{aligned}
$$

Condition (1a) has two meanings. The first is that $d_{i+1}-d_{i} \ll c T$. The radar rotation period is generally in the order of seconds, so condition (1a) is evidently valid. The second is that $\Delta t_{i+1, i} \ll T$, which is usually satisfied in the radar because the TOA measurement noise is typically on the order of $n s$ and the radar radiation source period is typically on the order of $s$.

The meaning of condition (2a) is that the angle $\theta_{i+1, i}$ between two measurements of the UAV and target is less than $0.5 \Pi$, so that the integer operation can be avoided to estimate $\left|K_{i+1, i}\right|= \pm i$, where $i$ is an integer. The rotation angle period of most target radars is $2 \pi$; that is, $\Pi=2 \pi$. When a UAV does not move radially relative to the target, we will have that $\theta_{i+1, i}<\pi=0.5 \Pi$, which satisfies the conditions.

It can be seen from conditions (1a) and (2a) that when the radar rotation period is known, $K$ can be accurately estimated.

4.1.2. Calculation Conditions with the Radar Rotation Period Estimated. The radar antenna rotation period is unknown 


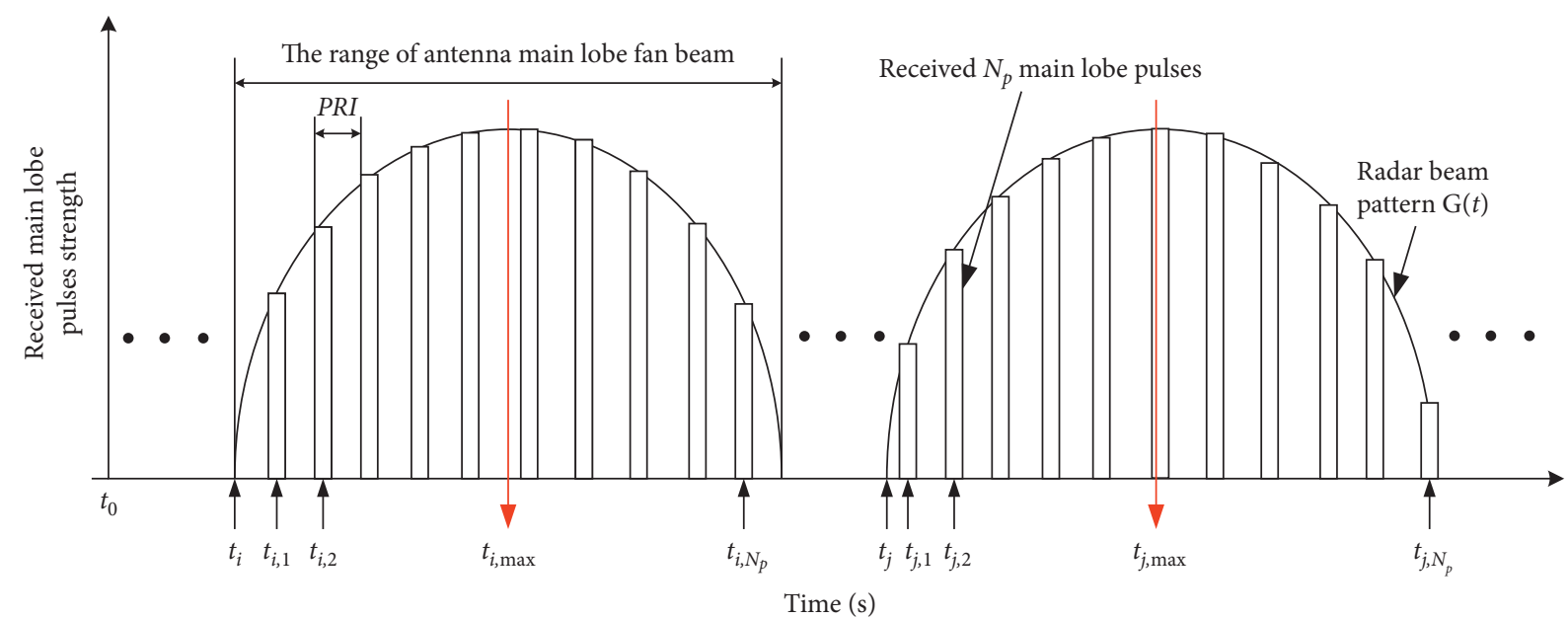

FIgURE 3: Received signal by UAV.

but can be estimated by the received radiation source pulse. It can be recorded as $\widehat{T}=T+\Delta T$, and $\Delta T$ is the estimation error. We have

$$
K_{i+1, i}=\operatorname{round}\left(\frac{\left(t_{i+1}-t_{i}\right)}{\widehat{T}}\right) .
$$

The integral fraction expansion is as follows:

$$
\begin{aligned}
\frac{t_{i+1}-t_{i}}{\widehat{T}} & =\frac{d_{i+1}-d_{i}}{c(T+\Delta T)}+\frac{\theta_{i+1, i}}{\Omega(T+\Delta T)}+\frac{K_{i+1, i} T}{T+\Delta T}+\frac{\Delta t_{i+1, i}}{T+\Delta T} \\
& =\frac{1}{(1+(\Delta T / T))}\left(\frac{d_{i+1}-d_{i}}{c T}+\frac{\theta_{i+1, i}}{\Pi}+K_{i+1, i}+\frac{\Delta t_{i+1, i}}{T}\right) .
\end{aligned}
$$

According to equation (19), the sufficient conditions for accurately estimating $K_{i+1, i}$ are as follows:

$$
\begin{aligned}
& \text { Condition (1b): }\left(\left(d_{i+1}-d_{i}\right) /(c T)\right)+\left(\left(\Delta t_{i+1, i}\right) / T\right) \approx 0 \\
& \text { Condition (2b): } \theta_{i+1, i}<0.5 \Pi \\
& \text { Condition }(3 \mathrm{~b}):(\Delta T / T) \approx 0
\end{aligned}
$$

The meaning of conditions (1b) and (2b) is consistent with conditions (1a) and (2a); the meaning of condition ( $3 b$ ) is that the estimation deviation of the rotation period of the radar antenna is negligible compared to the period itself, which is also the basic condition for the accuracy of the period estimation.

4.2. Observability Conditions of the Localization Model. The NVTDOA equation of the target radar position is nonlinear. In most papers, the observability theorem of Lee and Dum [25] is used to solve the observability condition of the equation. When the theorem is applied to discrete nonlinear systems, the equivalent conclusion is to judge the rank of the Gramer matrix. For a nonlinear system as shown in equation (20), let the system set be $S$ and the Gramer matrix of the state variables $x_{i 0}^{*}$ in the set is as in equation (21):

$$
\left\{\begin{array}{l}
x(n)=f[x(n), n] ; x(1)=x_{0} \\
y(n)=h(x(n), n)
\end{array}\right.
$$

$\boldsymbol{\Gamma}\left(i_{0}, i_{0}+N-1\right)=\left[\mathbf{H}_{i_{0}}, \mathbf{H}_{i_{0}+1} \boldsymbol{\Phi}_{i_{0}, i_{0}+1}, \ldots, \mathbf{H}_{i_{0}+N-1} \boldsymbol{\Phi}_{i_{0}, i_{0}+N-1}\right]^{T}$,

where $\mathbf{H}_{i}=(\partial y(n) / \partial x(n))$, which is the Jacobian matrix, and $\Phi$ is a state transition matrix. If there is an integer $N$ such that $\operatorname{rank} \Gamma(i, i+N-1)=n$ (state dimension), then the system is completely observable for $S$ [26].

4.2.1. Observability Analysis with the Radar Rotation Period Known. When the radar period is known, the state transition matrix $\boldsymbol{\Phi}_{i, i+1}=I_{2 \times 2}$ and the measurement equation of the variable $\mathbf{X}=\{D, L\}^{T}$ can be simplified as

$$
\operatorname{NVTDOA}_{i+1, i}=\left(\frac{\left(d_{i+1}-d_{i}\right)}{c}\right)+\left(\frac{\theta_{i+1, i}}{\Omega}\right)+K_{i+1, i} T+\Delta t_{i+1, i} .
$$

The Jacobi matrix of $\operatorname{NVTDOA}_{j, i}$ at $X$ is

$$
\mathbf{H}_{i}=\frac{\partial \operatorname{NVTDOA}_{i+1, i}}{\partial \mathbf{X}}=\left[\frac{\partial \operatorname{NVTDOA}_{i+1, i}}{\partial D}, \frac{\partial \operatorname{NVTDOA}_{i+1, i}}{\partial L}\right]^{\mathrm{T}} .
$$

From Figure 1 , it can be seen that $d_{i}=$ $\sqrt{D^{2}+L^{2}}$ and $d_{i+1}=\sqrt{D^{2}+\left(L-l_{i}\right)^{2}}$. By substituting $\theta_{i+1, i}=$ $\tan ^{-1}(L / D)-\tan ^{-1}\left(\left(L-l_{i}\right) / D\right)$ into equation (23), we can get

$$
\begin{aligned}
\mathbf{H}_{i}= & \frac{\partial \mathrm{NVTDOA}_{i+1, i}}{\partial \mathbf{X}} \\
= & {\left[\frac{1}{c}\left(\frac{D}{d_{i+1}}-\frac{D}{d_{i}}\right)+\frac{1}{\Omega}\left(\frac{L-l_{i}}{d_{i+1}^{2}}-\frac{L}{d_{i}^{2}}\right) \frac{1}{c}\left(\frac{L-l_{i}}{d_{i+1}}-\frac{L}{d_{i}}\right)\right.} \\
& \left.+\frac{1}{\Omega}\left(\frac{D}{d_{i}^{2}}-\frac{D}{d_{i+1}^{2}}\right)\right]^{\mathrm{T}} .
\end{aligned}
$$


Under far-field radiation source settings, the radar source is a two-dimensional target. Let the position of the UAV receiving the radiation source pulse be $S_{i}, S_{i+1}$, and $S_{i+2}$, and Gramer matrix based on NVTDOA by three measurements is

$$
\Gamma(i, i+2)=\left[\mathbf{H}_{i}, \mathbf{H}_{i+1}\right]^{\mathrm{T}},
$$

where

$$
\begin{aligned}
\mathbf{H}_{i+1}= & \frac{\partial \mathrm{NVTDOA}_{i+2, i+1}}{\partial \mathbf{X}} \\
= & {\left[\frac{1}{c}\left(\frac{D}{d_{i+2}}-\frac{D}{d_{i+1}}\right)+\frac{1}{\Omega}\left(\frac{L-l_{i}-l_{i+1}}{d_{i+2}^{2}}-\frac{L-l_{i}}{d_{i+1}^{2}}\right)\right.} \\
& \left.\cdot \frac{1}{c}\left(\frac{L-l_{i}-l_{i+1}}{d_{i+2}}-\frac{L-l_{i}}{d_{i+1}}\right)+\frac{1}{\Omega}\left(\frac{D}{d_{i+1}^{2}}-\frac{D}{d_{i+2}^{2}}\right)\right]^{\mathrm{T}} .
\end{aligned}
$$

The determinant of equation (25) is that

$$
\begin{aligned}
\operatorname{det} \Gamma(i, i+2)= & \frac{D}{c^{2}} \frac{M_{1}}{d_{i} d_{i+1} d_{i+2}}+\frac{D^{2}}{c \Omega} \frac{M_{2}}{d_{i}^{2} d_{i+1}^{2} d_{i+2}^{2}} \\
& +\frac{1}{c \Omega} \frac{M_{3}}{d_{i}^{2} d_{i+1}^{2} d_{i+2}^{2}}+\frac{D}{\Omega^{2}} \frac{M_{4}}{d_{i}^{2} d_{i+1}^{2} d_{i+2}^{2}},
\end{aligned}
$$

where

$$
\begin{aligned}
M_{1}= & l_{i+1}\left(d_{i+1}-d_{i}\right)-l_{i}\left(d_{i+2}-d_{i+1}\right), \\
M_{2}= & d_{i}^{2}\left(d_{i+2}-d_{i+1}\right)-d_{i+1}^{2}\left(d_{i+2}-d_{i}\right)+d_{i+2}^{2}\left(d_{i+1}-d_{i}\right), \\
M_{3}= & d_{i}^{2}\left(L-l_{i}-l_{i+1}\right)\left(L-l_{i}\right)\left(d_{i+2}-d_{i+1}\right) \\
& -d_{i+1}^{2} L\left(L-l_{i}-l_{i+1}\right)\left(d_{i+2}-d_{i}\right) \\
& +d_{i+2}^{2} L\left(L-l_{i}\right)\left(d_{i+1}-d_{i}\right), \\
M_{4}= & \left(d_{i+1}^{2}-d_{i+2}^{2}\right) l_{i}+\left(d_{i+1}^{2}-d_{i}^{2}\right) l_{i+1} .
\end{aligned}
$$

Discussions about $M_{i}$ are as follows:

(1) If $l_{i}=0$, then $M_{1} \neq 0$ when $l_{i+1} \neq 0$ and $d_{i} \neq d_{i+1}$. But $l_{i}=0$ and $d_{i} \neq d_{i+1}$ are contradictory, so this condition is not valid

(2) If $l_{i+1}=0$, then $M_{1} \neq 0$ when $l_{i} \neq 0$ and $d_{i+1} \neq d_{i+2}$. But $l_{i+1}=0$ and $d_{i+1} \neq d_{i+2}$ are contradictory, so this condition is not valid

Therefore, from (1) and (2), we can know $M_{1} \neq 0$ when $l_{i} \neq 0, l_{i+1} \neq 0$, and $d_{i} \neq d_{i+1} \neq d_{i+2}$

(3) When $d_{i} \neq d_{i+1} \neq d_{i+2} \neq 0, M_{2} \neq 0$

(4) If $l_{i}=0$, then $d_{i}=d_{i+1}$. So $M_{3}=0$. If $l_{i+1}=0$, then $d_{i+1}=d_{i+2}$. So $M_{3}=0$. Therefore, when $l_{i} \neq 0, l_{i+1} \neq 0$, and $d_{i} \neq d_{i+1} \neq d_{i+2}, M_{3} \neq 0$

(5) When $d_{i} \neq d_{i+1} \neq d_{i+2} \neq 0, l_{i} \neq 0$, and $l_{i+1} \neq 0, M_{4} \neq 0$

From (1)-(5) and (27), it can be seen that when $D \neq 0$, $d_{i} \neq d_{i+1} \neq d_{i+2} \neq 0, l_{i} \neq 0$, and $l_{i+1} \neq 0$, then $\operatorname{det} \Gamma(i, i+2) \neq 0$. It shows that $D$ and $L$ can be estimated by three NVTDOA measurements when UAV must move but not radially. So the target radar position can be determined.

4.2.2. Observability Analysis with the Radar Rotation Period Estimated. When the period $T$ is unknown, the period estimate $\mathbf{X}^{*}=\{D, L, T\}^{T}$ and the state transition matrix $\Phi_{i, i+1}=I_{3 \times 3}$ need to be added to analyze the observability. Without affecting observability, the period integer of two adjacent measurements of UAV is assumed to be 1 :

$$
\begin{aligned}
\mathbf{H}_{i}^{*} & =\frac{\partial \text { NVTDOA }_{i+3, i+2}}{\partial \mathbf{X}^{*}}=\left[\frac{1}{c}\left(\frac{D}{d_{i+1}}-\frac{D}{d_{i}}\right)+\frac{1}{\Omega}\left(\frac{L-l_{i}}{d_{i+1}^{2}}-\frac{L}{d_{i}^{2}}\right) \frac{1}{c}\left(\frac{L-l_{i}}{d_{i+1}}-\frac{L}{d_{i}}\right)+\frac{1}{\Omega}\left(\frac{D}{d_{i}^{2}}-\frac{D}{d_{i+1}^{2}}\right) 1\right]^{\mathrm{T}}, \\
\mathbf{H}_{i+1}^{*} & =\frac{\partial \mathrm{NVTDOA}_{i+3, i+2}}{\partial \mathbf{X}^{*}} \\
& =\left[\frac{1}{c}\left(\frac{D}{d_{i+2}}-\frac{D}{d_{i+1}}\right)+\frac{1}{\Omega}\left(\frac{L--l_{i}-l_{i+1}}{d_{i+2}^{2}}-\frac{L-l_{i}}{d_{i+1}^{2}}\right) \frac{1}{c}\left(\frac{L-l_{i}-l_{i+1}}{d_{i+2}}-\frac{L-l_{i}}{d_{i+1}}\right)+\frac{1}{\Omega}\left(\frac{D}{d_{i+1}^{2}}-\frac{D}{d_{i+2}^{2}}\right) 1\right]^{\mathrm{T}}, \\
\mathbf{H}_{i+2}^{*} & =\frac{\partial \mathrm{NVTDOA}_{i+3, i+2}}{\partial \mathbf{X}^{*}} \\
& =\left[\frac{1}{c}\left(\frac{D}{d_{i+3}}-\frac{D}{d_{i+2}}\right)+\frac{1}{\Omega}\left(\frac{L-l_{i}-l_{i+1}-l_{i+2}}{d_{i+3}^{2}}-\frac{L-l_{i}-l_{i+1}}{d_{i+2}^{2}}\right) \frac{1}{c}\left(\frac{L-l_{i}-l_{i+1}-l_{i+2}}{d_{i+3}}-\frac{L-l_{i}-l_{i+1}}{d_{i+2}}\right)+\frac{1}{\Omega}\left(\frac{D}{d_{i+2}^{2}}-\frac{D}{d_{i+3}^{2}}\right) 1\right]^{\mathrm{T}} .
\end{aligned}
$$


Similarly, the Gramer matrix based on NVTDOA by four measurements is

$$
\begin{aligned}
\Gamma^{*}(i, i+3)= & {\left[\mathbf{H}_{i}^{*}, \mathbf{H}_{i+1}^{*}, \mathbf{H}_{i+2}^{*}\right]^{\mathrm{T}}, } \\
\operatorname{det} \Gamma^{*}(i, i+3)= & \operatorname{det}\left(\left[\mathbf{H}_{i+1}, \mathbf{H}_{i+2}\right]\right)-\operatorname{det}\left(\left[\mathbf{H}_{i}, \mathbf{H}_{i+2}\right]\right) \\
& +\operatorname{det}\left(\left[\mathbf{H}_{i}, \mathbf{H}_{i+1}\right]\right) .
\end{aligned}
$$

From the calculation in the previous section, we can see the following:

(1) When $D \neq 0, d_{i} \neq d_{i+1} \neq d_{i+2} \neq 0, l_{i} \neq 0$, and $l_{i+1} \neq 0$, then $\operatorname{det}\left(\left[\mathbf{H}_{i}, \mathbf{H}_{i+1}\right]\right) \neq 0$

(2) When $D \neq 0, \quad d_{i+1} \neq d_{i+2} \neq d_{i+3} \neq 0, \quad l_{i+1} \neq 0$, and $l_{i+2} \neq 0$, then $\operatorname{det}\left(\left[\mathbf{H}_{i+1}, \mathbf{H}_{i+2}\right]\right) \neq 0$

(3) When $D \neq 0, \quad d_{i} \neq d_{i+2} \neq d_{i+3} \neq 0, \quad l_{i+1}+l_{i} \neq 0$, and $l_{i+2} \neq 0$, then $\operatorname{det}\left(\left[\mathbf{H}_{i}, \mathbf{H}_{i+2}\right]\right) \neq 0$

In summary, the observability conditions of equation (30) can be obtained; that is, $D \neq 0$ and $d_{i} \neq d_{i+1} \neq d_{i+2} \neq d_{i+3} \neq 0$, $l_{i} \neq 0, l_{i+1} \neq 0, l_{i+2} \neq 0$. That is to say, $D, \mathrm{~L}$, and $T$ can be estimated by four NVTDOA measurements when UAV must move but not radially. So the target radar position can be determined.

\section{Construction and Solution of NVTDOA Localization Equation Based on Cuckoo Search}

5.1. Construction of NVTDOA Localization Equation. When the UAV moves, we have

$$
\Psi_{j, i}=t_{j}-t_{i}
$$

The value of $\Psi_{j, i}$ can be estimated by Section 3.2. Referring to equation (13), it can be concluded that

$$
\Psi_{j, i} \approx\left(\frac{\left(d_{j}-d_{i}\right)}{c}\right)+\left(\frac{\theta_{j, i}}{\Omega}\right)+K_{j, i} T,
$$

where $K_{j, i}=\operatorname{round}\left(\Psi_{j, i} / T\right)$. The distance from $S_{i}$ to $S_{0}$ is $d_{i}$ at $t_{i}$ time, the distance from $S_{j}$ to $S_{0}$ is $d_{j}$, and the distance from $S_{j}$ to $S_{i}$ is $l_{i}$.

It should be noted that from the conclusion of Section 4.2 , at low speed the observability condition of the model is related to the change of the position of the UAV and has nothing to do with its speed. If the position of the UAV is recorded during the movement, the error will be caused because the initial time $t_{i}$ is not well determined. In order to locate $S_{i}$ accurately, the UAV hovers approximately before $t_{i}$ to obtain the exact position of the main lobe of the receiving radar antenna during the $i$ th scanning period. After receiving the main lobe pulse in this period, the UAV flies to position $S_{j}$ until receiving the main lobe pulse in the $j$ th scanning period of the radar antenna.

As shown in Figure 1, the geometric relationship diagram of periodic emitter location based on NVTDOA is obtained. For convenience of representation, let $S_{i+1}=S_{j}$ and $S_{i+2}=S_{l}$. In the Cartesian rectangular two-dimensional coordinate system, the radar emitter is located at $S_{0}=\left[x_{0}, y_{0}\right]^{T}$, UAV is located at $S_{i}=\left[x_{i}, y_{i}\right]^{T}, S_{j}=\left[x_{j}, y_{j}\right]^{T}$, and $S_{l}=\left[x_{l}, y_{l}\right]^{T}$. It is known from the Beidou localization system that its error can be ignored. Based on the accurate estimation of $\Psi_{j, i}$, equation (33) can be reduced to

$$
\Psi_{j, i} \approx\left(\frac{\left(d_{j}-d_{i}\right)}{c}\right)+\left(\frac{\theta_{j, i}}{\Omega}\right)+K_{j, i} T \approx\left(\frac{\theta_{j, i}}{\Omega}\right)+K_{j, i} T,
$$

where $\quad \theta_{j, i}=\cos ^{-1}\left(\left(d_{i}^{2}+d_{j}^{2}-l_{i}^{2}\right) / 2 d_{i} d_{j}\right), \quad d_{i}=$ $\sqrt{\left(x_{i}-x_{0}\right)^{2}+\left(y_{i}-y_{0}\right)^{2}}, \quad d_{j}=\sqrt{\left(x_{j}-x_{0}\right)^{2}+\left(y_{j}-y_{0}\right)^{2}}$, $l_{i}=\sqrt{\left(x_{j}-x_{i}\right)^{2}+\left(y_{j}-y_{i}\right)^{2}}$, and $\Omega=(\Pi / T)$. The radar system rotation period angle is $\Pi=360^{\circ}$. The approximation method used in the equation is the same as condition (1a) in Section 4. Substituting $d_{i}, d_{j}$, and $l_{i}$ into $\theta_{j, i}$, we have

$$
\theta_{j, i}=\cos ^{-1}\left(\frac{\left(x_{0}-x_{i}\right)\left(x_{0}-x_{j}\right)+\left(y_{0}-y_{i}\right)\left(y_{0}-y_{j}\right)}{\sqrt{\left(x_{0}-x_{i}\right)^{2}+\left(y_{0}-y_{i}\right)^{2}} \cdot \sqrt{\left(x_{0}-x_{j}\right)^{2}+\left(y_{0}-y_{j}\right)^{2}}}\right) .
$$

Substituting equation (35) into equation (34) yields

$$
\Psi_{j, i} \cdot \Omega-K_{j, i} \cdot \Pi-\cos ^{-1}\left(\frac{\left(x_{0}-x_{i}\right)\left(x_{0}-x_{j}\right)+\left(y_{0}-y_{i}\right)\left(y_{0}-y_{j}\right)}{\sqrt{\left(x_{0}-x_{i}\right)^{2}+\left(y_{0}-y_{i}\right)^{2}} \cdot \sqrt{\left(x_{0}-x_{j}\right)^{2}+\left(y_{0}-y_{j}\right)^{2}}}\right)=0 .
$$

Equation (36) constructs a nonlinear equation based on the position $S_{0}=\left[x_{0}, y_{0}\right]^{T}$ of the radar radiation source.
Similarly, when the UAV flies from $S_{i}=\left[x_{j}, y_{j}\right]^{T}$ to $S_{l}=\left[x_{l}, y_{l}\right]^{T}$, there will be

$$
\Psi_{l, j} \cdot \Omega-K_{l, j} \cdot \Pi-\cos ^{-1}\left(\frac{\left(x_{0}-x_{j}\right)\left(x_{0}-x_{l}\right)+\left(y_{0}-y_{j}\right)\left(y_{0}-y_{l}\right)}{\sqrt{\left(x_{0}-x_{j}\right)^{2}+\left(y_{0}-y_{j}\right)^{2}} \cdot \sqrt{\left(x_{0}-x_{l}\right)^{2}+\left(y_{0}-y_{l}\right)^{2}}}\right)=0 .
$$


By solving the nonlinear equations consisting of equations (36) and (37), the localization results can be obtained that $\widehat{S}_{0}=\left[\widehat{x}_{0}, \widehat{y}_{0}\right]^{T}$.

\subsection{Solution of Nonlinear Localization Equations Based on Cuckoo Search Algorithm}

5.2.1. Cuckoo Search Algorithm. The cuckoo search (CS) algorithm is generated by the simulation study of cuckoo's nesting behavior by Yang Xin-she and DEB Suash. The individuals in birds flocking select nests by Levy flight and abandon by discovered probability $P_{a}$, so the birds population is updated. The paths and locations of cuckoo nests are updated as follows:

$\mathbf{x}_{i}^{t+1}=\mathbf{x}_{i}^{t}+\alpha_{0}\left(\mathbf{x}_{i}^{t}-\mathbf{x}_{\text {best }}^{t}\right) \oplus \operatorname{Levy}(\lambda), \quad i=1,2, \ldots, n$,

where $\mathbf{x}_{i}^{t}=\left(x_{i, 1}^{t}, \ldots, x_{i, d}^{t}\right)^{T}$ is the $i$ th solution of the $t$ th generation, $\alpha_{0}=0.01$, $\mathbf{x}_{\text {best }}^{t}$ is the optimal solution of the $t$ th generation, $\operatorname{Levy}(\lambda) \sim \phi \times u /|v|^{1 / \lambda}$ is the dot product, $n$ is the number of nests, and $\operatorname{Levy}(\lambda) \sim \phi \times u /|v|^{1 / \lambda}$ is the Levy random search path whose moving step size obeys levy probability distribution as

$$
\operatorname{Levy}(\lambda) \sim \frac{\phi \times u}{|v|^{1 / \lambda}}
$$

where $u$ and $v$ follow normal distribution and $\phi=\left((\Gamma(1+\lambda) \sin (\lambda \pi / 2)) /\left(\Gamma(1+\lambda / 2) \times 2^{(1+\lambda / 2)} \lambda\right)\right)$, where $\Gamma$ is the standard Gamma function.

Combining equations (38) and (39), in CS algorithm, the new solution $\mathbf{x}_{i}^{t+1}$ is generated by

$$
\mathbf{x}_{i}^{t+1}=\mathbf{x}_{i}^{t}+0.01\left(\mathbf{x}_{i}^{t}-\mathbf{x}_{\text {best }}^{t}\right) \oplus \frac{\phi \times u}{|v|^{1 / \lambda}}, \quad i=1,2, \ldots, n .
$$

After discarding a part of solutions according to the discovery probability, a new solution is generated by

$$
\mathbf{x}_{i}^{t+1}=\mathbf{x}_{i}^{t}+r\left(\mathbf{x}_{j}^{t}-\mathbf{x}_{e}^{t}\right),
$$

where $r$ is the random number of uniform distribution in $(0$, 1) interval and $\mathbf{x}_{j}^{t}$ and $\mathbf{x}_{e}^{t}$ are random solutions of the $t$ th generation.

5.2.2. Solution of Nonlinear Localization Equations Based on Cuckoo Search Algorithm. The localization equations consisting of equations (36) and (37) are recorded as

$$
\left\{\begin{array}{l}
f_{1}(x, y)=0 \\
f_{2}(x, y)=0
\end{array}\right.
$$

When equation (42) is transformed into the unconstrained optimization problem, the objective function of the cuckoo search algorithm is

$$
F(\mathbf{w})=\sum_{i=1}^{2}\left(f_{i}(\mathbf{w})\right)^{2}
$$

where $\mathbf{w}=(x, y)^{T}$. So solving equation (42) is to find the minimum solution of equation (43).

To sum up, the solution process of CS is as follows:
TABLE 1: Main performance parameters of the radar AN/SPS49.

\begin{tabular}{lc}
\hline Parameters & Index \\
\hline Frequency & $f_{c}=850 \sim 942 \mathrm{MHz}$ \\
Transmission power & Peak $P_{s}=360 \mathrm{~kW} ;$ \\
Sweep azimuth of radar antenna & $\Omega=360^{\circ}$ \\
Azimuth rotation rate & $\Omega=36^{\circ} / \mathrm{s}$ \\
$3 \mathrm{~dB}$ azimuth beamwidth of radar pulse & $\theta_{3}=3.3^{\circ}$ \\
signal & $\mathrm{PRF}=1000 \mathrm{~Hz}$ \\
Radar pulse repetition frequency & $\Delta \theta=\Omega / \mathrm{PRF}=0.036^{\circ}$ \\
Rotation angle resolution & $N_{p}=\theta_{3} / \Delta \theta=91.6$ \\
Number of received main beam pulses in & \\
one radar sweep cycle & $\tau=125 \mu \mathrm{s}$ \\
Radar pulse length &
\end{tabular}

TABLE 2: Main performance parameters of UAV and receiving loads.

\begin{tabular}{lc}
\hline Parameters & Index \\
\hline Receiver sampling frequency & $f_{s}=2 \mathrm{MHz}$ \\
UAV speed & $v=30 \mathrm{~m} / \mathrm{s}$ \\
Initial position of UAV & $S_{i}=[0,0]^{T}$ \\
Error variance of UAV position measurement & $\sigma_{s}^{2}=0 \mathrm{~m}$ \\
Main valve pulse receiving interval time & $\Delta T_{i, j}=60 \mathrm{~s}$ \\
Receiving pulse SNR & $\mathrm{SNR}=20 \mathrm{~dB}$ \\
\hline
\end{tabular}

(1) Initialization. The population number of the cuckoo algorithm is $m$, the maximum number of iterations is $K$, and the discovered probability is $P_{a}$. Moreover, $m$ nest positions are randomly generated and the optimal nest position of the current generation, $\mathbf{w}_{0 \text {,best }}$ (best $\in\{1,2, \ldots, m\}$ ), and the optimal fitness value, $F_{\min }$, are found. In the simulation experiment of the paper, $m=20, K=2000$, and $P_{a}=0.25$.

(2) Update of Levy Flight Path (Loop Body). The optimal value of the previous generation, $\mathbf{w}_{k-1 \text {,best }}$, is reserved. $K$ represents the current evolutionary generation. And a new nest location is generated by equation (40). Then we compare the optimal fitness value of the new nest location with that of the previous nest location and keep the solution with the smaller optimal fitness value which is expressed as $\mathbf{I}_{k}=\left[\mathbf{w}_{k, 1}, \mathbf{w}_{k, 2}, \ldots, \mathbf{w}_{k, m}\right]$ to the next nest location.

(3) Update of the Discovered Probability (Loop Body). For each individual in $\mathbf{q}_{k}=\left[\mathbf{w}_{k, 1}, \mathbf{w}_{k, 2}, \cdots, \mathbf{w}_{k, m}\right], r$ is randomly generated as the probability that the bird's egg is found by the nest owner, that is, the discovered probability. If $r<P_{a}$, the nest value is unchanged; otherwise, the nest is updated according to equation (41). The new nest is compared with $\mathbf{q}_{k}=$ $\left[\mathbf{w}_{k, 1}, \mathbf{w}_{k, 2}, \cdots, \mathbf{w}_{k, m}\right]$, and then the better solution which is expressed as $\mathbf{q}_{k}=\left[\mathbf{w}_{k, 1}, \mathbf{w}_{k, 2}, \ldots, \mathbf{w}_{k, m}\right]$ is kept to the next generation.

(4) Judgment Body. The optimal nest position $\mathbf{w}_{k \text {,best }}$ in $\mathbf{q}_{k}$ and the fitness value $F_{\min }$ of the solution are calculated. At the same time, it is determined whether the termination condition of the algorithm (the generation is equal to $K$ ) is satisfied. If it is satisfied, the global 


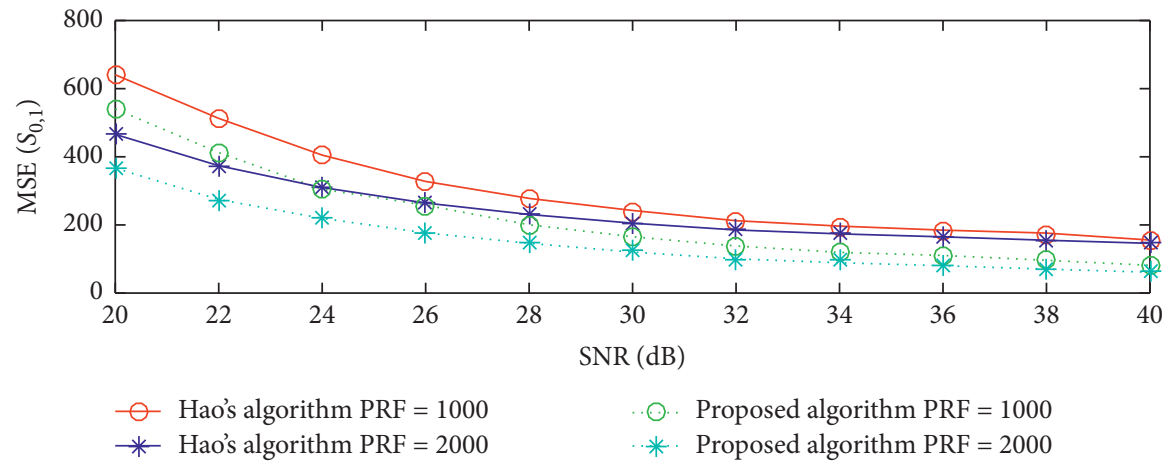

(a)
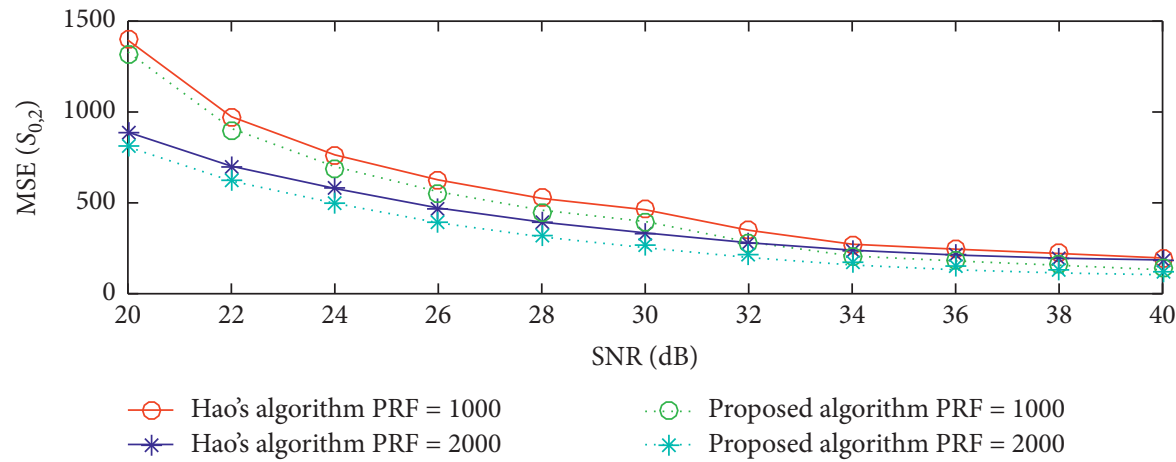

(b)

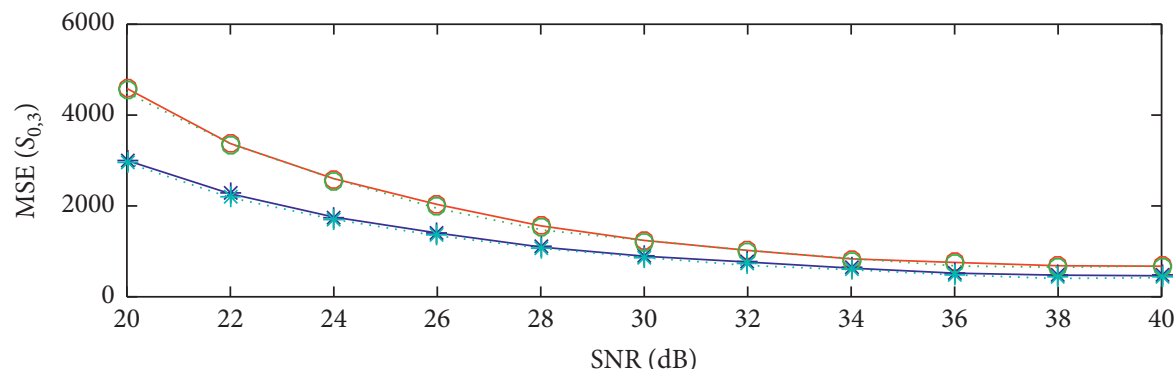

- Hao's algorithm PRF $=1000$

๑. Proposed algorithm $\mathrm{PRF}=1000$

* Hao's algorithm PRF $=2000$

*. Proposed algorithm PRF $=2000$

(c)

FIgURE 4: Estimation accuracy performance as the received SNR increases with different PRFs.

optimal solution is obtained. Otherwise, step 2 is returned, and the iterative operation continues until the termination condition is satisfied.

\section{Simulation and Performance Analysis}

6.1. Simulation Parameter Setting. The American Thunder AN/SPS49 air-to-air search radar is taken as the simulation object in this paper. The main parameters of the radar are shown in Table 1.

The horizontal pattern of the antenna main lobe beam is of Gaussian type, and the classical mathematical model as equation (44) is used in the simulation:

$$
G(t)=\exp \left[-4 \ln \sqrt{2}\left(\frac{\Omega}{\theta_{3}}\right)^{2} t^{2}\right] .
$$

Without loss of generality, the main performance parameters of UAV and receiving loads are set in Table 2.

In this section, the localization accuracy for the radar antenna by the above algorithm is verified. The localization accuracy is calculated by $\operatorname{MSE}\left(S_{0}\right)=\sum_{l=1}^{L}\left\|\widehat{S}_{0}^{l}-\mathbf{S}_{0}\right\|^{2} / L$, where $\hat{S}_{0}^{l}$ is the estimated value of the lth Monte Carlo simulation of the radar antenna localization, $L$ is the number of simulation, and 1000 is selected here.

6.2. Sensitivity of $\mathbf{S}_{0}$ Estimation to Pulse Repetition Frequency and Received SNR. The radar pulse energy received by UAV is inversely proportional to the quadratic of $d_{i}$. According to the radar average power, there will also be a higher SNR. Therefore, the radar radiation source is simulated at different positions, and the three target positions are selected as 
$S_{0,1}=[60,80]^{T} \mathrm{~km}, \quad S_{0,2}=[90,90]^{T} \mathrm{~km}, \quad$ and $\quad S_{0,3}=$ $[160,145]^{T} \mathrm{~km}$. The type of noise, as discussed in Section 4 , is additive random noise with the mean of 0 and the variance of $\sigma_{\xi}^{2}$.

Let SNR be monotonically increasing in the interval of $[20,40] \mathrm{dB}$. When the radar pulse repetition frequency is $\mathrm{PRF}=1000 \mathrm{~Hz}, \mathrm{PRF}=2000 \mathrm{~Hz}$, the pulse signal time width is $\tau=125 \mu \mathrm{s}$, the UAV speed is $v=50 \mathrm{~m} / \mathrm{s}$, UAV flight time for the single localization $\Delta T_{j, i}=\Delta T_{j, l}=60 \mathrm{~s}$, and the localization of three radars are $S_{0,1}=[60,80]^{T} \mathrm{~km}$, $S_{0,2}=[90,90]^{T} \mathrm{~km}$, and $S_{0,3}=[160,145]^{T} \mathrm{~km}$. Three radars are positioned separately. That is, the UAV receives only one radar signal at a time, and there is no signal aliasing. In order to verify the accuracy and effectiveness of the proposed algorithm, Hao's method in [21] is compared because Hao's method and the method in this paper both give the solution of the VTDOA localization algorithm. MSE $\left(S_{0}\right)$ with different SNRs and PRFs is shown in Figure 4.

As shown in Figure 4, with the increase of SNR, the localization accuracy of Hao and the proposed algorithm also become higher. When the noise power becomes smaller, the influence of $\Delta t_{i+1, i}$ becomes smaller, which is more in line with the neglect condition of $\Delta t_{i+1, i}$. So the localization accuracy is higher. When the PRF is increased, the UAV can obtain more useful information in the same time. So the localization accuracy is higher. As shown in Figure 4, with the increase of PRF, MSE $\left(S_{0}\right)$ is reduced and the localization accuracy is higher, which is consistent with the theoretical analysis. By comparing and analyzing the simulation curve of the proposed algorithm and Hao's algorithm, it can be seen that the localization accuracy is improved under different PRFs and SNRs with the proposed algorithm in this paper. However, for the same target radar, $\mathrm{PRF}=1000 \mathrm{~Hz}$ and $\mathrm{PRF}=2000 \mathrm{~Hz}$, the proposed algorithm has little improvement compared with Hao's algorithm. This shows that with the increase of PRF, the improvement of accuracy with the proposed algorithm is not obvious compared with that of Hao's algorithm. This is the result of the estimation of $\Psi_{j, i}$ in this algorithm is the same as that in Hao's. However, with the difference of $d_{i}$, the improvement of Hao's accuracy will be different which will be analyzed and explained below.

When PRF $=2000 \mathrm{~Hz}$, the curves of localization accuracy of three radars versus SNR are shown in Figure 5. As shown in Figure 5, when PRF and SNR are the same, the MSE $\left(S_{0}\right)$ becomes lower as $d_{i}$ increases. Because as $d_{i}$ increases, SNB accepted by UAV increases. But the localization accuracy of this algorithm is higher than that of Hao's algorithm. With different $d_{i}$ the improvement of localization accuracy by the proposed algorithm compared with Hao's algorithm is shown in Figure 6. It can be seen that the improvement becomes better as $d_{i}$ decreases. So the proposed algorithm works better in the relatively close localization area, which is also the requirement of the small aircraft to detect the target at close range.

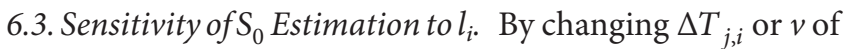
$\mathrm{UAV}$, the distance $l_{i}$ between two UAVs in a single measurement is changed, where $l_{i}=\Delta T_{j, i} v$. In the simulation,

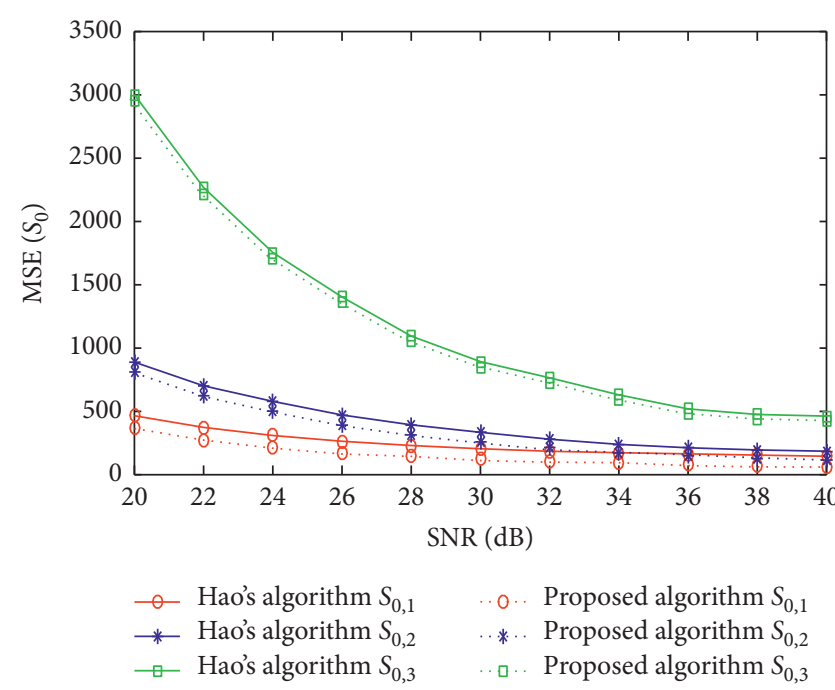

FIgURE 5: Localization accuracy with different $D_{i}$.

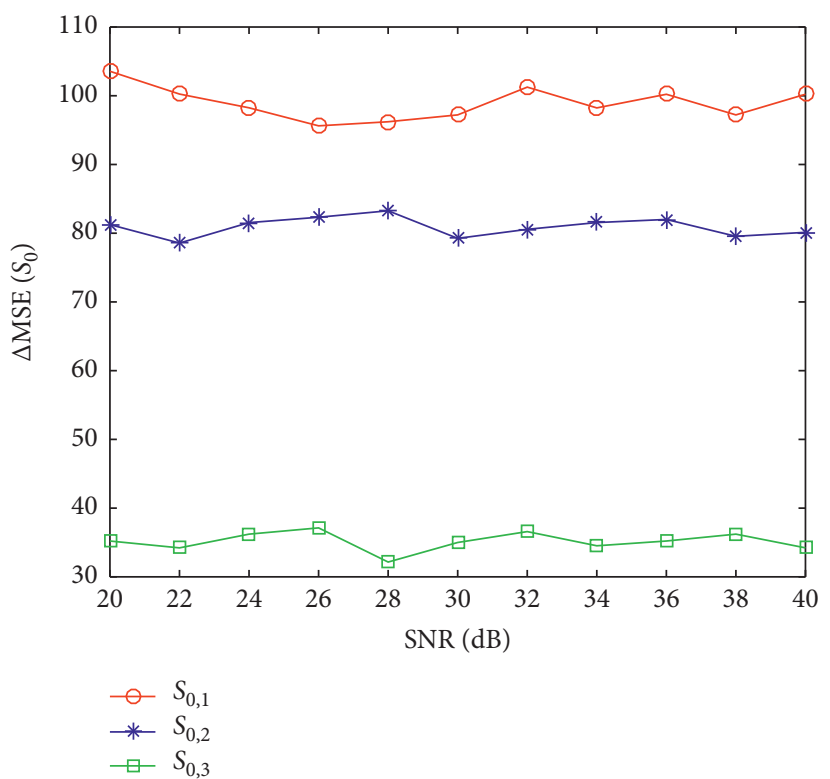

FIgURE 6: The improvement of localization accuracy with different $D_{i}$ by the proposed algorithm in this paper.

$v=50 \mathrm{~m} / \mathrm{s}$, which is kept unchanged, $l_{i}$ is changed by changing $\Delta T_{j, i}$. The accuracy varies with $\Delta T_{j, i}$ at $\mathrm{PRF}=1000 \mathrm{~Hz}, \mathrm{SNR}=30 \mathrm{~dB}$, and $\tau=125 \mu \mathrm{s}$ as shown in Figure 7 . When $\Delta T_{j, i}$ increases from $10 \mathrm{~s}$ to $150 \mathrm{~s}$, the localization accuracy of the three radars have the same trend. In Hao's algorithm, when $\Delta T_{j, i} \in[50 \mathrm{~s}, 100 \mathrm{~s}]$, the best localization performance is obtained. When $\Delta T_{j, i}<50 \mathrm{~s}$, with the increase of $\Delta T_{j, i}$, the accuracy is greatly improved. Because the aperture of the VTDOA localization system increases in a reasonable range, it will help to improve accuracy. But when $\Delta T_{j, i}>100 \mathrm{~s}$, the accuracy becomes worse. This is due to that the wavefront of radar signal received by UAV twice will not be the same plane wave because of the excessive $l_{i}$. Therefore, the calculation method of rotation time difference of the radar antenna in Hao's 


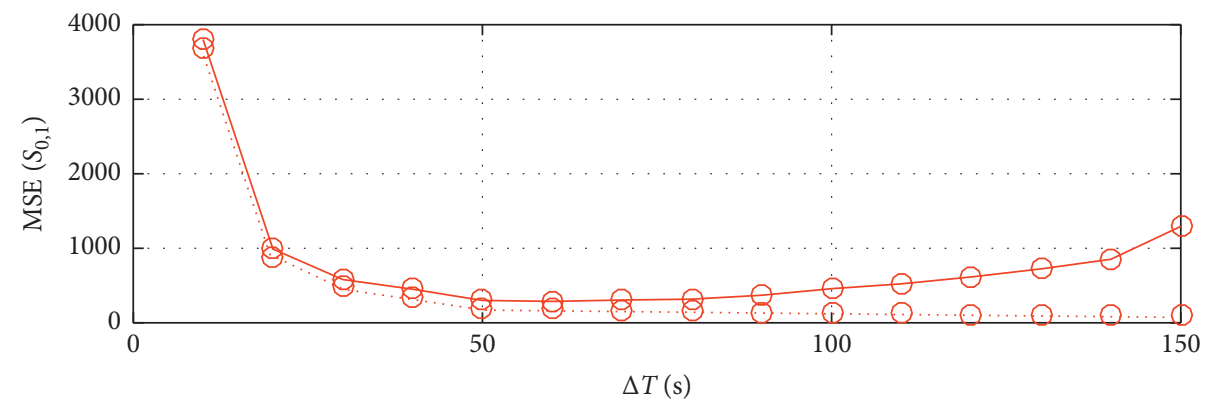

- Hao's algorithm

... Proposed algorithm

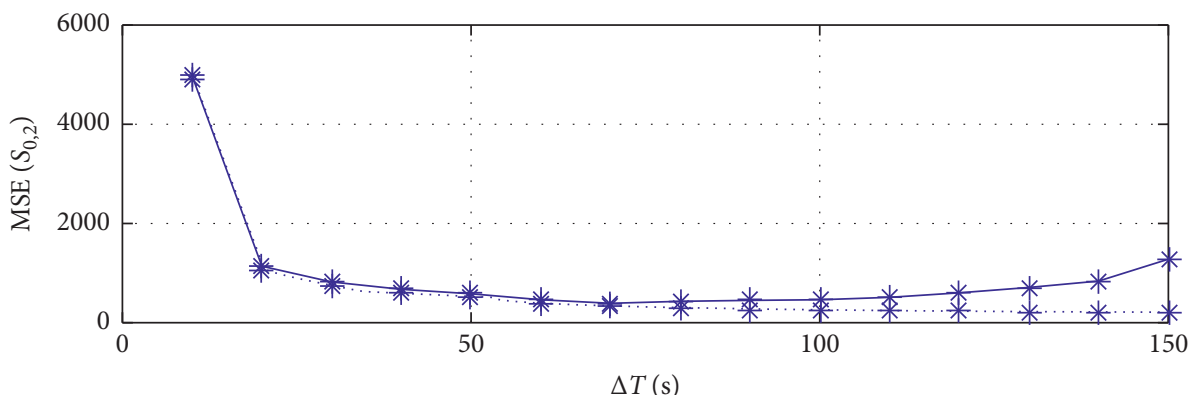

* Hao's algorithm

$\Delta T(\mathrm{~s})$

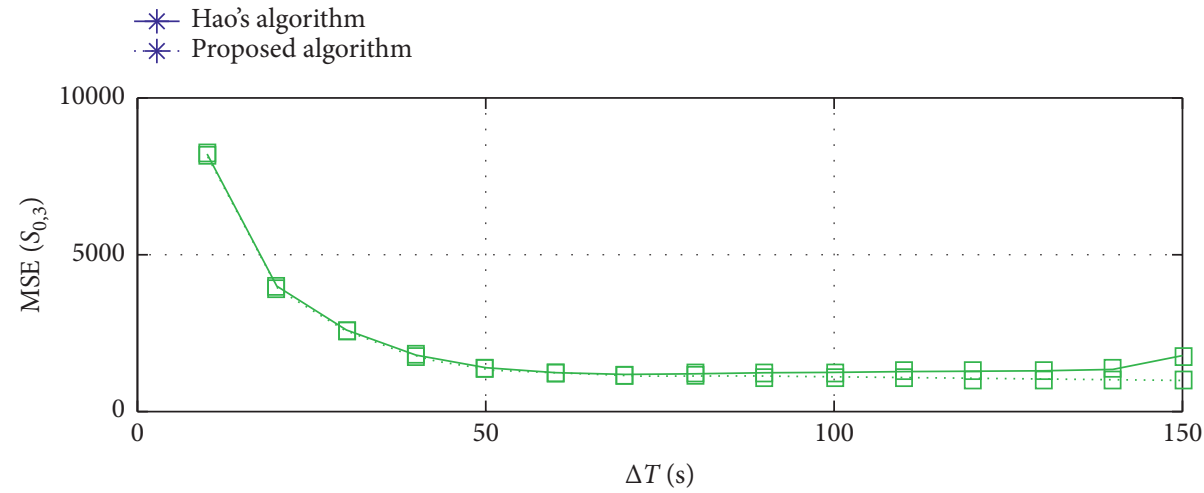

$\square$ Hao's algorithm

.๑. Proposed algorithm

FIgURE 7: Localization accuracy with $\Delta T$.

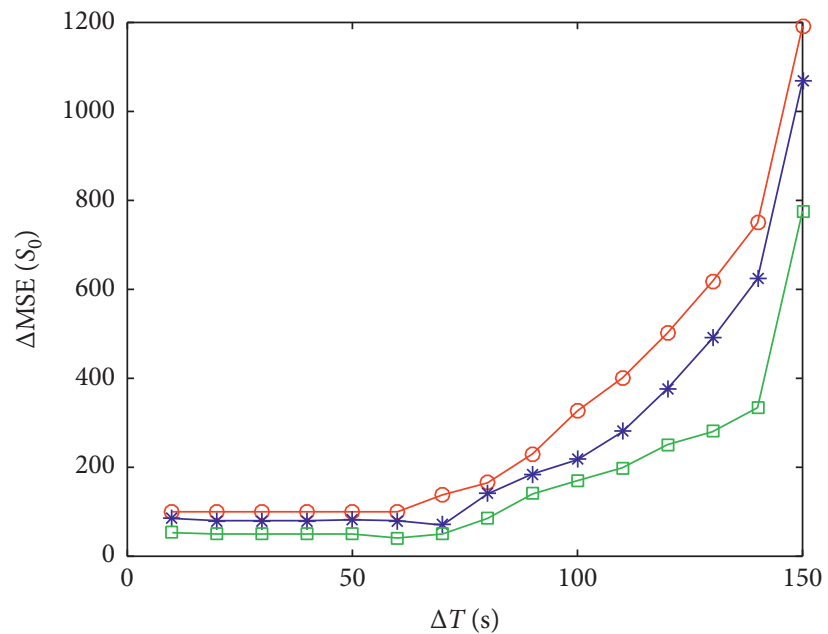

$$
\begin{aligned}
& \curvearrowleft S_{0,1} \\
& \rightarrow-S_{0,2} \\
& -\square S_{0,3}
\end{aligned}
$$

Figure 8: The improvement of localization accuracy with $\Delta T$ by the proposed algorithm in this paper. 
algorithm brings more errors. In this paper, the NVTDOA localization equations are proposed by using the improved rotation time difference of the radar antenna calculation method. The Newton iteration is used to obtain the convergence solution, which avoids the approximate calculation in Hao's method. And $\Delta \operatorname{MSE}\left(S_{0}\right)$ is not less than 100 .

By the proposed algorithm, with the increase of $\Delta T_{j, i}$, the accuracy increases, and there is no case that the accuracy deteriorates when $\Delta T_{j, i}$ is too large. In order to further illustrate the advantages of the proposed algorithm, the improvement of localization accuracy by the algorithm compared with Hao's algorithm is shown in Figure 8. With $\Delta T_{j, i}$ increasing, the accuracy increases, which is consistent with the above analysis. The accuracy with smaller $D_{i}$ is better, so the accuracy of close-range target detection by $\mathrm{UAV}$ is better improved in this paper.

\section{Conclusion}

When the distance between localization points of UAV is large, the localization error of the VTDOA algorithm increases. First, based on the shortcomings of VTDOA, the NVTDOA localization model of periodic radiation source based on single-station small-load UAV is established considering the integral characteristics of radar antenna rotation time difference. Second, the NVTDOA localization equation is constructed and the convergence solution of the NVTDOA localization equation is obtained by the Cuckoo search algorithm to realize the localization of the radar radiation source. Last, the simulation and comparative analysis of the influence of positioning distance, pulse repetition frequency, SNR, and UAV linear distance on location precision are carried out. The simulation results show that Cuckoo search NVTDOA can improve the positioning accuracy and some conclusions are as follows:

(1) Comparing the localization accuracy of three different position radars, we can see that, in the relatively close localization area, the improvement effect is better. So the NVTDOA algorithm has higher practical value in the close-range target detection by UAV.

(2) With different SNRs, the localization accuracy of NVTDOA is better than that of VTDOA. Therefore, NVTDOA has stronger noise robustness.

(3) The simulation experiments of different motion time of the UAV are carried out. The results show that the smaller the linear distance between two positioning of UAV is, the larger the localization error of the VTDOA algorithm is. But localization accuracy of NVTDOA has little dependence on UAV localization distance, which shows that NVTDOA is more suitable for localization of UAV with high speed and long motion time.

\section{Data Availability}

The data used to support the findings of this study are available from the corresponding author upon request.

\section{Conflicts of Interest}

The authors declare that they have no conflicts of interest.

\section{Acknowledgments}

This work was supported in part by the National Natural Science Foundation of China (Grant no. 61601496).

\section{References}

[1] M. Malanowski and K. Kulpa, "Two methods for target localization in multistatic passive radar," IEEE Transactions on Aerospace and Electronic Systems, vol. 48, no. 1, pp. 572-580, 2012.

[2] A. Canclini, F. Antonacci, A. Sarti, and S. Tubaro, "Acoustic source localization with distributed asynchronous microphone networks," IEEE Transactions on Audio, Speech, and Language Processing, vol. 21, no. 2, pp. 439-443, 2013.

[3] R. M. Buehrer, H. Wymeersch, and R. M. Vaghefi, "Collaborative sensor network localization: algorithms and practical issues," Proceedings of the IEEE, vol. 106, no. 6, pp. 1089-1114, 2018.

[4] D. He, Y. Zhang, and W. Yu, "Research on location technology of wireless communication system based on nonlinear multi-base station distributed chaotic stochastic resonance method," Navigation, Position and Timing, vol. 6, no. 2, pp. 58-66, 2019.

[5] S. S. Dias and M. G. S. Bruno, "Cooperative target tracking using decentralized particle filtering and RSS sensors," IEEE Transactions on Signal Processing, vol. 61, no. 14, pp. 36323646, 2013.

[6] Z. Liu, D. Hu, Y. Zhao, and Y. Zhao, "An algebraic method for moving source localization using TDOA, FDOA, and differential doppler rate measurements with receiver location errors," EURASIP Journal on Advances in Signal Processing, vol. 2019, no. 1, p. 25, 2019.

[7] H. Benjian, L. Wang, L. Zan, and Y. Zhao, "Sensor selection method for TDOA passive localization," Journal of Electronics and Information Technology, vol. 41, no. 2, pp. 462-468, 2019.

[8] T. Slavisa, B. Marko, and T. Milan, "A linear estimator for network localization using integrated RSS and AOA measurements," IEEE Signal Processing Letters, vol. 26, no. 3, pp. 405-409, 2019.

[9] Z. Tan, X. Shi, and Y. Xu, "AOA positioning algorithm based on RSS curve of second degree fitting," Application Research of Computers, vol. 34, no. 6, pp. 1854-1857, 2017.

[10] Y. Wang and K. C. Ho, "TDOA positioning irrespective of source range," IEEE Transactions on Signal Processing, vol. 65, no. 6, pp. 1447-1460, 2017.

[11] E. J. Walsh, "Analysis of experimental NRL radar altimeter data," Radio Science, vol. 9, no. 8-9, pp. 711-722, 1974.

[12] J. Xu, Z. Wang, Q. Liu, and L. Ren, "A novel TOA estimation method for unknown signal based on intra-pulse correlation accumulation," in Proceedings of the International Conference on Signal Processing, IEEE Nanded, India, October 2016.

[13] Y. T. Chan, B. H. Lee, R. Inkol, and F. Chan, "Estimation of pulse parameters by convolution," in Proceedings of the Canadian Conference on Electrical \& Computer Engineering, IEEE, Ottawa, Canada, May 2006.

[14] P. Wan, Research and Application on the Key Technologies for TDOA Passive Source Localization, Xidian University, Xi'an, China, 2018. 
[15] O. Nickens and M. Darko, "Emitter geolocation with two uavs," in Proceedings of the Conference on Information, Decision and Control, IEEE, Adelaide, Australia, February 2007.

[16] D. H. Kim, K. Lee, M. Y. Park, and J. Lim, "UAV-based localization scheme for battlefield environments," in Proceeding of the IEEE Military Communications Conference, IEEE, San Diego, CA, USA, November 2013.

[17] M. N. Sousa and R. S. Thomä, "Localization of UAV in urban scenario using multipath exploiting TDoA fingerprints," in Proceeding of the IEEE 29th Annual International Symposium on Personal, Indoor and Mobile Radio Communications (PIMRC), pp. 1394-1399, IEEE, Bologna, Italy, September 2018.

[18] Y. Liu and Y. Shen, "UAV-aided high-accuracy relative localization of ground vehicles," in Proceedings of the IEEE international conference on communications (ICC), pp. 1-6, IEEE, Kansas City, MO, USA, May 2018.

[19] Y. Dalveren and A. Kara, "Multipath exploitation in emitter localization for irregular terrains," Radioengineering, vol. 27, no. 2, pp. 473-482, 2019.

[20] M. N. Sousa and R. S. Thomä, "Single sensor RF emitter location using ray tracing multipath exploitation," in Proceedings of the 15th international symposium on wireless communication systems (ISWCS), pp. 1-6, IEEE, Lisbon, Portugal, August 2018.

[21] B. Hao, L. Tong, Z. Li, S. Xiao, and L. Gu, "Passive radar source localisation based on PSAAA using single small size aircraft," IET Radar, Sonar \& Navigation, vol. 10, no. 7, pp. 1191-1200, 2016.

[22] J. Zhu, C. Yue, H. Benjian, G. Niu, and P. Wan, "Passive radar source localization using synthetic aperture antenna array," Acta Electronica Sinica, vol. 45, no. 10, pp. 2332-2336, 2017.

[23] C. Jauffret and D. Pillon, "Observability in passive target motion analysis," IEEE Transactions on Aerospace and Electronic Systems, vol. 32, no. 4, pp. 1290-1300, 1996.

[24] A. N. Payne, "Observability problem for bearings-only tracking," International Journal of Control, vol. 49, no. 3, pp. 761-768, 1989.

[25] T. S. Lee, K. P. Dunn, and C. B. Chang, "On observability and unbiased estimation of nonlinear systems," System Modeling and Optimization, Springer, Berlin, Germany, 1982.

[26] Y. Liu, Research on Novel Methods for Time-Based Source Localization Using Moving Observers, National University of Defense Technology, Changsha, China, 2016. 